\title{
IMAGING SPECTROSCOPY OF FLOWS IN ACTIVE REGIONS
}

\author{
P. MEIN, N. MEIN \\ Observatoire de Paris, Section de Meudon, F92195 Meudon Cedex
}

\begin{abstract}
We use imaging spectroscopy from the MSDP of the VTT telescope in Tenerife (collaboration Paris-Meudon Observatory and Kiepenheuer Institut) to analyse Doppler velocities of arch filaments in $\mathrm{H}_{\mathrm{a}}$ and $\mathrm{NaD_{1 }}$. The geometry of the magnetic loops is deduced from perspective effects with a simple dynamical model. Velocities are correlated in both lines.

Further results are expected from simultaneous observations with other instruments (SVST of La Palma and Huairou magnetograph).
\end{abstract}

\section{COORDINATED OBSERVATIONS}

On May 7, 1991, arch filaments have been observed in AR6615 (S10-W26). Several instrumemnts were involved (see also the poster by $\mathrm{N}$. Mein et al., "Active region evolution through coordinated observations"):

- Multichannel Subtractive Double Pass spectrograph of the VTT telescope at Tenerife (Paris-Meudon Observatory and Kiepenheuer Institut), providing simultaneous 2D pictures in two line profiles: 9 channels in $H_{\alpha}$ and 11 in $N a D_{1}$ (P. Mein, 1991).

- Swedish telescope (SVST) at La Palma, providing high resolution pictures in white light

- Magnetographs (Huairou, GCT at Tenerife)

- SERTS experiment (EUV spectra and spectroheliograms)

\section{FLOWS IN $H_{\alpha}$ ARCH FILAMENTS}

Several arch filaments cross the magnetic neutral line. They exhibit strong doppler shifts in $H_{\alpha}$. If the structures are not optically thick, the corresponding velocities underestimate the real material velocities (Alissandrakis et al., 1990). But we can assume that the ratios of doppler shifts at both feet $V_{A} / V_{B}$ are close to the ratios of material velocities. The observed values are typically $\mathbf{- 0 . 2}$ (small blueshifts in the direction of disk centre, large redshifts in the opposite direction).

The angle $\phi$ between the direction of disk centre and the line joining the feet is roughly $40^{\circ}$, and the distance to the centre is defined by $\theta=27^{\circ}$. 


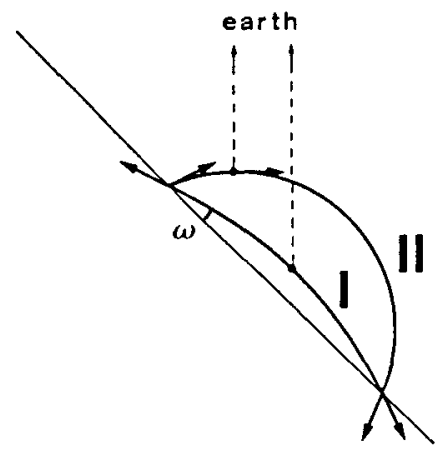

FIGURE I Models I (free fall) and II (siphon) providing the same ratio of dopplershifts at the feet of the arches.

\section{DYNAMICAL MODELS AND GEOMETRY}

We assume that the matter is flowing along magnetic loops, the shape of which is circular in vertical planes (with respect to the sun). The angle $\omega$ defines the inclination of the loops at the feet (figure I).

We test two possible dynamical models:

Model (I): equal downflows in both legs of the arches (example: free fall)

Model (II): equal upflow and downflow (example: siphon flow)

Taking into account the values of $\theta, \phi$ and $V_{A} / V_{B}$, we get $\omega \simeq 17^{0}$ for model (I), and $\omega \simeq 30^{\circ}$ for model (II).

There is a way to discriminate between models (I) and (II). The location of the zero dopplershift point is

- roughly in the middle part of the arch in model (I) (real zero velocity of material)

- close to the foot which points to disk centre in model (II) (velocity perpendicular to the line of sight).

The observations are clearly in favour of model (I).

We conclude that $\omega \simeq 17^{\circ}$, and that the tops of the loops are roughly $3000 \mathrm{~km}$ above the chromosphere.

\section{CORRELATIONS BETWEEN $H_{\alpha}$ AND $D_{1}$ DOPPLERSHIFTS}

Some preliminary results have been obtained by correlating dopplershifts observed simultaneously in both lines. By restricting the field of analysis to the absorbing $H_{\alpha}$ structures, we obtain results such as the histogram of figure (II).

Some correlation does exist. The ratio between line-of-sight velocities should be roughly 10 if the $H_{\alpha}$ arches are optically thick, and more if they are thin. 


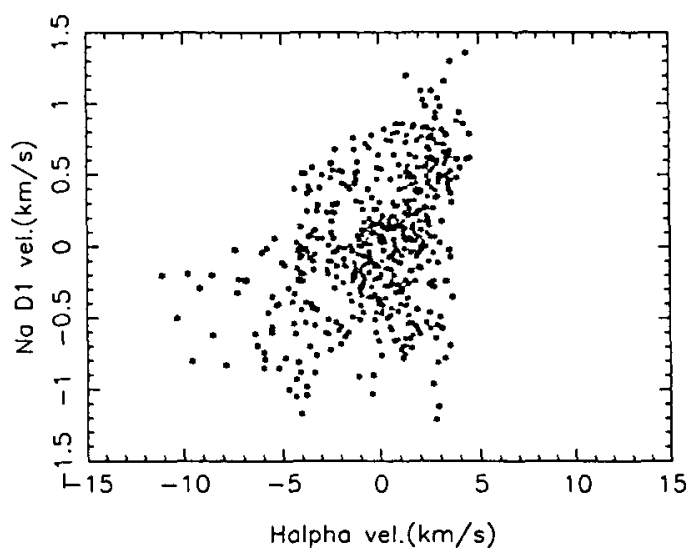

FIGURE II Histogram of doppler velocities in photosphere $(\mathrm{NaD})$ and chromosphere $\left(H_{\alpha}\right)$.

This ratio can be used as a constraint on MHD models of the loops. It is connected with the scale-heights of the density and the magnetic field along the loops.

\section{CONCLUSION AND PERSPECTIVES}

The MSDP imaging spectroscopy in two lines simultaneously, is very efficient for 3D observations of mass flows. We deduced simple geometrical models as well as constraints on density and magnetic field scale-heights.

Further developments might be: fields

- the comparison of the loop geometry with the extrapolation of magnetic

- the comparison of the evolution of velocity fields with the horizontal flows derived from SVST observations (white light).

\section{ACKNOWLEDGMENTS}

We thank C. Coutard, R. Hellier, F. Colson, C. Brechet and the staff of the VTT for help in the observations. The images were processed by the microdensitometer MAMA of INSU (CNRS).

\section{REFERENCES}

Alissandrakis C.E., Tsiropoula G., Mein P. 1990, Astron. Astrophys. 230, 200. Mein P. 1991, Astron. Astrophys. 248, 669. 